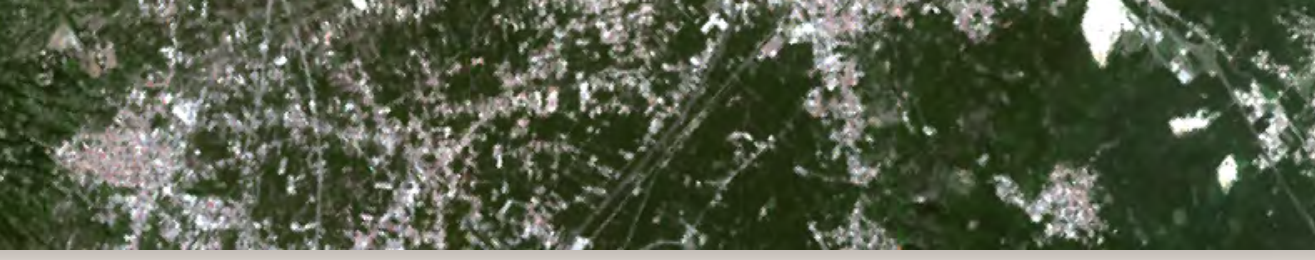

\title{
Landsat Surface Reflectance Data
}

Landsat satellite data have been produced, archived, and distributed by the U.S. Geological Survey (USGS) since 1972. Users rely on these data for historical study of land surface change and require consistent radiometric data processed to the highest science standards. In support of the guidelines established through the Global Climate Observing System, the USGS has embarked on production of higher-level Landsat data products to support land surface change studies. One such product is Landsat surface reflectance.

Surface reflectance data products approximate what a sensor held just above the Earth's surface, without any artifacts from the atmosphere, illumination, and viewing geometry, would measure. The removal of atmospheric artifacts increases the consistency and comparability between images of the Earth's surface taken at different times. Many higher-level geospatial products-including vegetation indexes, albedo, Leaf Area Index (LAI), burned area, land cover, and land cover change - rely on surface reflectance products.

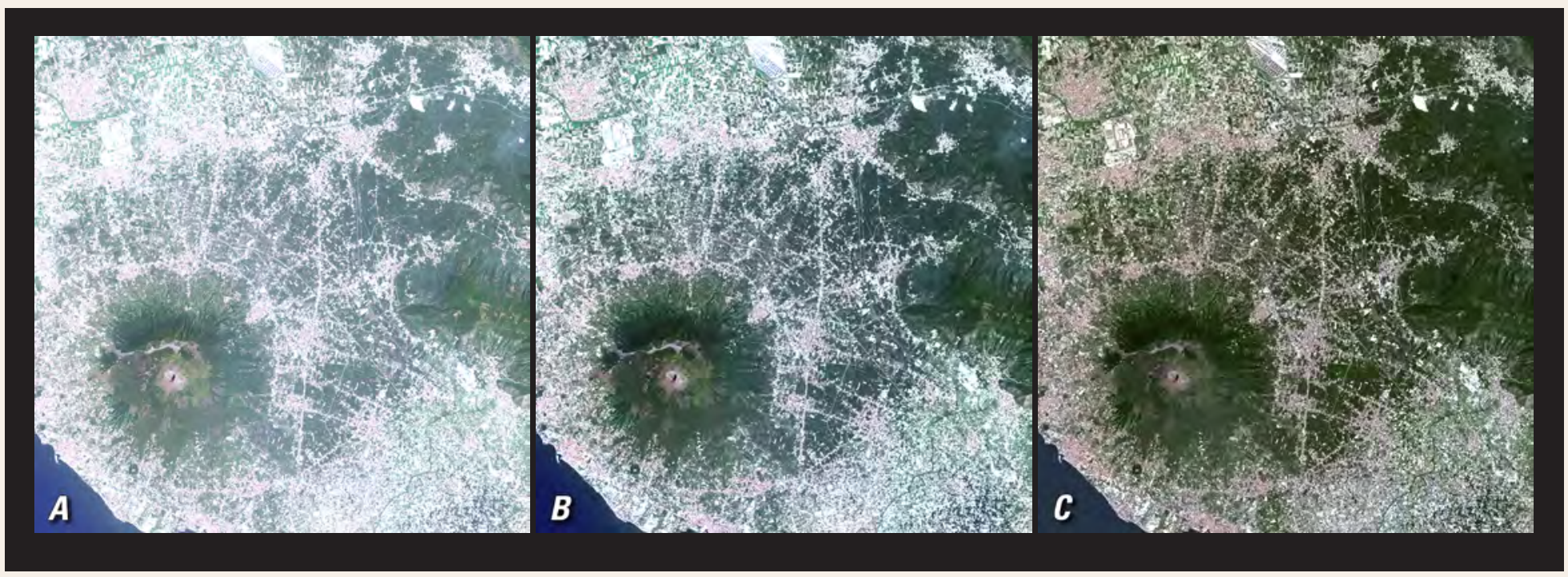

These subsets of a Landsat scene of Mount Vesuvius near Naples, Italy, display $A$, natural color composite; $B$, top-of-atmosphere reflectance; and C, surface reflectance processing. (Source: Landsat 8 Operational Land Imager [OLI] Path 189 Row 32, acquired May 29, 2013.)

Surface reflectance and other high level data products can be requested through the USGS Earth Resources Observation and Science (EROS) Center by accessing the EROS Science Processing Architecture (ESPA) interface at: https://espa.cr.usgs. gov/.

Surface reflectance data are also available using EarthExplorer (https://earthexplorer.usgs.gov), under the "Landsat" datasets.

All Landsat Level 1 data that meet the following criteria are accepted for surface reflectance processing:

- Landsat 8: Most Operational Land Imager (OLI) scenes acquired from April 11, 2013, to within 1 week of present acquisitions

- Landsat 7: All Enhanced Thematic Mapper Plus (ETM+) scenes acquired from 1999 to within 1 week of present acquisitions
- Landsat 4-5: Most Thematic Mapper (TM) scenes acquired from August 1982 to May 2012. Some Landsat 4-5 TM scenes are formatted and calibrated differently, and cannot be processed to surface reflectance. These scenes will be automatically removed from submitted orders.

Landsat surface reflectance data support land surface change studies and are considered provisional. More information on surface reflectance can be accessed at https://www.usgs.gov/ land-resources/nli/landsat/landsat-surface-reflectance.

Questions regarding surface reflectance data can be directed to:

Landsat Customer Services

USGS EROS

Sioux Falls, SD 57198

(605) 594-6151

Email: custserv@usgs.gov 